\title{
Comparative Study on the Effect of Anticoagulants in the Incidence of Lower Limb DVT in Sleeved Patients with Low BMI
}

\author{
Mohab G. Elbarbary, MD; Ahmed S. M. Omar, MD; Ahmed A. Khalil, MD, FACS \\ Department of General Surgery, Faculty of Medicine, Ain shams University, Egypt
}

Background: Venous thromboembolism (VTE) is a common postoperative complication after bariatric surgery with high mortality. Our study aimed to spotlight on the importance of anticoagulants after sleeve gastrectomy and its effect on lower limb deep venous thrombosis (DVT) incidence in obese patients with low BMI.

Patients and methods: A prospective observational study was performed, including 200 patients with BMI between 35 and $45 \mathrm{~kg} / \mathrm{m} 2$ undergoing laparoscopic sleeve gastrectomy (LSG) in the period between February 2017 and September 2018. Half of the patients received anti-thromboembolic dosage scheme of $0.5 \mathrm{mg} / \mathrm{kg} / \mathrm{day}$ with the induction of anaesthesia and for one week postoperatively (Group A) while the other half had no anticoagulation therapy (Group B).

Results: Patients were followed with lower limb venous duplex at 2 weeks, 3 and 6 months interval from the operation or on appearance of symptoms of DVT. No patients had DVT in both groups by venous duplex.

Conclusion: The incidence of lower limb DVT after LSG in patients with low BMI is not affected only by anticoagulants. We need further investigations including coagulation profile and congenital anticoagulation abnormalities to give an accurate idea about the influence of LSG on the incidence of DVT.

Keywords: Laparoscopic sleeve gastrectomy, deep venous thrombosis, anticoagulants.

\begin{abstract}
Introduction
The etiology of venous thrombosis involves mainly three factors which were referred to as Virchow's triad. Those simply were damage to the vessel wall, venous stasis, and hypercoagulability. ${ }^{1}$
\end{abstract}

Recent advances in coagulation and vascular biology had redefined the importance of Virchow's model. Not all components of Virchow's triad were of equal importance in all patients. ${ }^{2}$

It had been concluded that deep venous thrombosis arises from the interaction of multiple inherited and acquired risk factors. This complex interaction (referred to as "thrombotic potential"), had been promoted by Rosendaal. He had realized that when the cumulative thrombotic potential of all risk factors exceeds a certain threshold, clinical thrombosis will probably occur. ${ }^{3}$ This theory was supported by the observation that the risk of DVT increases with the number of risk factors present. ${ }^{4}$ The commonest site for deep vein thrombi is in the calf, with common sites of origin in the Soleal sinusoids and behind the venous valves. ${ }^{5}$

Morbid and truncal obesity were identified as major predisposing factors for VTE. Sedentary lifestyle, increased abdominal pressure and excessive weight resting on the inferior vena cava drainage attributed to the increased risk. Additional risk factors include advanced age, history of previous VTE, immobilization, venous insufficiency and stasis, smoking, estrogen containing oral contraceptives and hormone replacement therapy, hypercoaguable state, hypoventilation syndrome, and anastomotic leakage. According to current literature, obesity interferes in intrinsic and extrinsic coagulation pathways, as well as in the anticoagulant mechanism, leading to a hypercoagulable state. Plasma concentration of fibrinogen, Von Willebrand factor, TPA, PAI-1 and factor VII are significantly elevated in obese patients, while platelet aggregation is promoted due to leptin. There is evidence that treatment of morbid obesity can reverse partially some of the above abnormalities, as weight loss is associated with significant reduction in fibrinogen, TPA, PAI-1 and improvement of deficiency of antithrombin III. ${ }^{6}$

More specifically, in laparoscopic bariatric surgery, reverse Trendelenburg position and pneumoperitoneum are associated with venous stasis of lower extremity and impaired venous return due to the compression of iliac veins and inferior vena cava. Severe obesity was associated with a large number of comorbidities. These started at the head (stroke, diabetic retinopathy, pseudo tumor cerebri, tinnitus) and went to the toes 
(diabetic neuropathy, venous stasis disease, foot ulcers) and affected almost every organ in between: heart, lungs, liver, gall bladder, spleen, esophagus, intestines, colon, kidneys, bladder, ovaries, prostate, breast, legs, etc. ${ }^{7}$

Bariatric surgery has demonstrated to be the most effective and sustainable method for the regulation of morbid obesity, superior to both pharmaceuticals and combinations of diet and lifestyle regimens.

In 1991 the National Institutes of Health published a consensus statement regarding bariatric surgery. Surgery was indicated in patients with a BMI $\geq$ $40 \mathrm{~kg} / \mathrm{m} 2$ and in patients with a BMI between 35 and 40 with other comorbidities. Severe sleep apnea, obesity related cardiomyopathy, Pickwickian syndrome, severe diabetes mellitus and lifestyle limitations were all considered comorbidities that would allow the patient to pursue surgery. ${ }^{8}$

LSG has increased in popularity and is currently very popular among laparoscopic surgeons involved in bariatric surgery. As LSG proved to be effective in achieving considerable weight loss in the shortterm, it has been proposed by some as a sole bariatric procedure. ${ }^{9}$

\section{Patients and methods}

This prospective observational study was carried out in Ain Shams University hospitals. An informed consent was obtained from the patients for participation in this study. This assessment was approved by the Ethical Committee of the Faculty of Medicine, Ain Shams University.

Our study included 200 morbidly obese patients underwent LSG in the period between February 2017 and September 2018. The patients were chosen by single blinded closed envelope method.

The age of the patients was $\geq 19$ years with a preoperative BMI 35 or $35-40 \mathrm{~kg} / \mathrm{m} 2$ with comorbidities (Hypertension, Dyslipidemia, Type 2 DM, sleep apnea, etc...) or with a preoperative BMI $40-45 \mathrm{~kg} / \mathrm{m} 2$ with or without co-morbidities.

\section{Then the study population was divided into two groups: \\ Group A (100 patients): This group was subjected to only chemical thromboprophylaxis in the form of clexane $(0.5 \mathrm{mg} / \mathrm{kg}$ IU $/$ day $)$ S.C and postoperative clexane $(0.5 \mathrm{mg} / \mathrm{kg}$ IU /day) for 1 week and early ambulation was encouraged.}

Group B (100 patients): This group was not subjected to any chemical or mechanical thromboprophylaxis and early ambulation was encouraged.
Inclusion criteria: All morbid obese patients undergoing Laparoscopic sleeve gastrectomy with BMI of $35-45 \mathrm{~kg} / \mathrm{m} 2$.

Exclusion criteria: Documented congenital / acquired coagulation disorders, concomitant anticoagulant for other risk factors, hypersensitivity to heparin and derivatives, history of recent or old thromboembolism, patient not compliant to therapy, primary deep venous reflux, duplex proven deep venous abnormalities e.g. congenital stenosis, patients that didn't tolerate fluid intake in the early postoperative period, bed ridden patients or limitations of self-dependant ambulation, and patients with BMI above $45 \mathrm{~kg} / \mathrm{m} 2$.

\section{Steps of the study}

Preoperative: Patients were evaluated by:

- Full history taking, including Co-morbidities e.g. DM, HTN, IHD, varicose veins, hyperlipidemia, old CVS.

- Measurement of the weight, height, and calculating BMI.

- Routine laboratory studies as ECG, CXR, CBC, RBS, ALT, S.Cr, and PT, PTT, INR.

- Venous duplex in patients with history of previous DVT.

- All patients were informed about surgical technique and risks of the operation with instructions for the importance of early ambulation after surgery and sufficient fluid intake.

Surgery: All patients underwent LSG under general anesthesia, complete aseptic conditions and good intra operative hydration with I.V fluids.

Postoperative: All patients were encouraged for early ambulation within two hours after recovery and were subjected to good hydration in hospital stay and after discharge. All patients were discharged on clear fluids intake for 10 days duration and were followed up postoperative by history of any complaints eg, LL pain or swelling or sudden attack of dyspnea, clinical examination. All patients had done Duplex venous scan in both Lower Limbs at 2 weeks, 3 months and 6 months post- operatively.

\section{Statistical analysis}

Data were collected, revised, coded and entered to the Statistical Package for Social Science (SPSS) version 20 and the following were done:

Qualitative data were presented as number and percentages while quantitative data were presented as mean, standard deviations and ranges.

The comparison between two groups with qualitative data were done by using Chi-square test and/or Fisher exact test was used instead of Chi-square test when the expected count in any cell was found 
less than 5.

The comparison between two independent groups with quantitative data and parametric distribution was done by using Independent t-test.

Pearson correlation coefficients were used to assess the relation between two studied parameters in the same group.

Receiver operating characteristic curve was used to assess the best cut off point with its sensitivity, specificity, positive predictive value and negative predictive value.

The confidence interval was set to $95 \%$ and the margin of error accepted was set to $5 \%$. So, the $p$-value was considered \pm significant as the following: $P>0.05$ : Non-significant. $P<0.05$ : Significant. $\mathrm{P}<0.01$ : Highly significant.

\section{Results}

Table 1: Demographic data

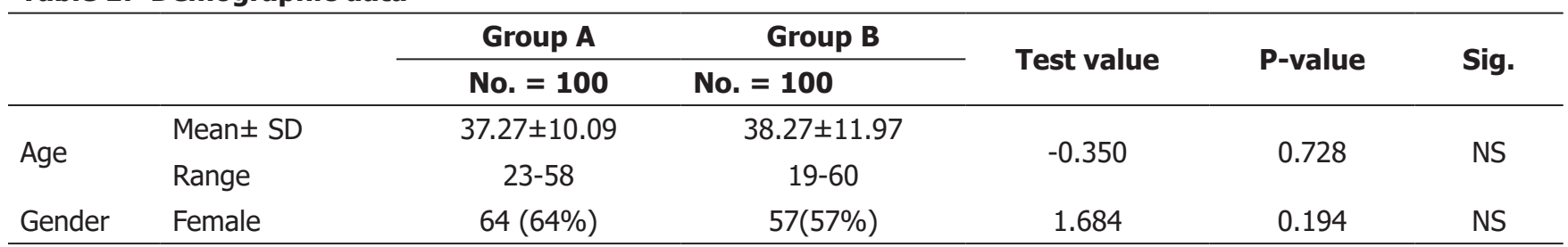

Table 2: Comparison between the two groups in weight, BMI and operative time

\begin{tabular}{|c|c|c|c|c|c|c|}
\hline & & Group A & Group B & \multirow{2}{*}{ Test value } & \multirow{2}{*}{ P-value } & \multirow{2}{*}{ Sig } \\
\hline & & No. $=100$ & No. $=100$ & & & \\
\hline Weight & Mean \pm SD & $112.5 \pm 12.8$ & $114.2 \pm 13.6$ & -0.913 & 0.362 & NS \\
\hline BMI & Mean \pm SD & $41.5970 \pm 2.08547$ & $41.0230 \pm 2.42790$ & 1.793 & 0.074 & NS \\
\hline \multicolumn{7}{|l|}{ Operative } \\
\hline time (min) & Mean \pm SD & $43.8500 \pm 6.947$ & $44.9100 \pm 5.29550$ & -1.213 & 0.226 & NS \\
\hline
\end{tabular}

Table 3: Comparison of incidence of DVT in both groups

\begin{tabular}{|c|c|c|c|c|c|c|c|c|}
\hline \multirow{2}{*}{ No. } & & \multicolumn{2}{|c|}{ Group A } & \multicolumn{2}{|c|}{ Group B } & \multirow{2}{*}{$\begin{array}{c}\text { Test } \\
\text { value }\end{array}$} & \multirow{2}{*}{ P-value } & \multirow{2}{*}{ Sig. } \\
\hline & & $\%$ & No. & $\%$ & & & & \\
\hline \multirow{2}{*}{2 weeks post operative Duplex LL } & Negative & 100 & $100.0 \%$ & 100 & $100.0 \%$ & \multirow{2}{*}{ NA } & \multirow{2}{*}{ NA } & \multirow{2}{*}{ NA } \\
\hline & Positive & 0 & $0.0 \%$ & 0 & $0.0 \%$ & & & \\
\hline \multirow{2}{*}{3 months post operative Duplex LL } & Negative & 100 & $100.0 \%$ & 100 & $100.0 \%$ & \multirow{2}{*}{ NA } & \multirow{2}{*}{ NA } & \multirow{2}{*}{ NA } \\
\hline & Positive & 0 & $0.0 \%$ & 0 & $0.0 \%$ & & & \\
\hline \multirow{2}{*}{6 months post operative Duplex LL } & Negative & 100 & $100.0 \%$ & 100 & $100.0 \%$ & \multirow{2}{*}{ NA } & \multirow{2}{*}{ NA } & \multirow{2}{*}{ NA } \\
\hline & Positive & 0 & $0.0 \%$ & 0 & $0.0 \%$ & & & \\
\hline
\end{tabular}




\section{Discussion}

Despite current VTE prevention methods, VTE continues to be an important source of postoperative morbidity and mortality among patients undergoing bariatric surgery. ${ }^{10}$

In the modern era of bariatric surgery with majority of programs having VTE prophylaxis protocols in place, the incidence of symptomatic DVT and PE ranges from $0 \%-5.4 \%$ and $0 \%-6.4 \%$, respectively. A recent systematic review of 19 studies evaluating VTE after laparoscopic bariatric surgery reported an incidence of pulmonary embolism of $0.5 \%$, and the Michigan Bariatric Surgery Collaborative published 2 large series from their quality collaborative registry that showed overall VTE rates less than $0.5 \%$ in average risk bariatric patients. Accurate evidencebased risk assessment tools for VTE in bariatric patients are currently not available, but a lot of literatures highlight several risk factors that must be taken into consideration when determining a prophylaxis strategy. These risk factors may include prior VTE, higher body mass index (BMI), age, gender, immobility, use of hormone therapy, obesity hypoventilation syndrome, pulmonary hypertension, venous stasis disease, operative time, and procedure type and approach. ${ }^{11}$

Data published in 2009 from the multicenter prospective Longitudinal Assessment of Bariatric Surgery (LABS) study reported a 30-day incidence of VTE complications of $0.4 \%$ and the risk increased with increasing weight. More recently, LABS data were used to determine whether prophylactic anticoagulation added to compression devices could prevents VTE. The overall 30-day VTE rate among 4416 patients was low $(0.25 \%$ among patients receiving sequential compression alone [ $n=396]$ and $0.47 \%$ when anticoagulation was added [ $\mathrm{n}=4020]$ ). This study concluded that there was insufficient evidence to make a specific recommendation regarding VTE prophylaxis after bariatric surgery and that a sufficiently powered trial to answer this question is impractical. ${ }^{12}$

In our study we reveal that DVT was not affected by laparoscopic sleeve gastrectomy if the patient had no coagulation system affection and had appropriate surgery with optimum operation time with early postoperative ambulation and good perioperative hydration.

\section{Conclusion}

There is no difference in DVT incidence between patients receiving chemoprophylaxis and those not receiving any chemoprophylaxis before and after LSG as the incidence of lower limb DVT in patients with low BMI is not affected only by anticoagulants. We need further investigations including coagulation profile and congenital anticoagulation abnormalities to give an accurate idea about the influence of laparoscopic sleeve gastrectomy on the incidence of thromboembolic events.

\section{References}

1. Kearon C: A conceptual framework for two phases of anticoagulant treatment of venous thrombo-embolism. J Thromb Haemost. 2012; 10: 507-511.

2. Bulger $\mathrm{CM}$, Jacobs $\mathrm{C}$, Patel $\mathrm{NH}$ : Epidemiology of acute deep vein thrombosis. Techniques in Vascular and Interventional Radiology. 2004; 7(2): 50-54.

3. Rosendaal FR, Roach REJ, Lijfering WM, van Hylckama Vlieg A, Helmerhorst FM, Rosendaal $F R$, et al: The risk of venous thrombosis in individuals with a history of superficial vein thrombosis and acquired venous thrombotic risk factors. Blood. 2013; 122: 4264-4269.

4. CushmanM, GlynnRJ, Goldhaber SZ, MollS, Bauer $K A$, Deitcher $S$, et al: Hormonal factors and risk of recurrent venous thrombosis: the prevention of recurrent venous thromboembolism trial. $\boldsymbol{J}$ Thromb Haemost. 2006; 4: 2199-2203.

5. Anderson FA, Wheeler HB, Goldberg RJ, et al: A population-based perspective of the hospital incidence and case-fatality rates of deep vein thrombosis and pulmonary embolism. Arch Intern Med. 1991; 151: 933-938.

6. Eleni Z, Eleni S, George T, Dimitris Z: Venous Thromboembolism in bariatric surgery. Pulmonary Embolism. 2012: 67-74.

7. Sugerman HJ, Sugerman EL, DeMaria EJ, et al: Bariatric surgery for severely obese adolescents. J Gastrointest Surg. 2003; 7: 102-108.

8. National institutes of health: Managing Overweight and Obesity in Adult. U.S Department of Health and Human Services. 2013.

9. Iannelli $A$, Dainese $R$, Piche $T$, Facchiano $E$, Gugenheim J: "Laparoscopic sleeve gastrectomy for morbid obesity." World J Gastroenterol. 2008; 14(6): 821-827.

10. Sapala JA, Wood MH, Schuhknecht MP, Sapala MA: Fatal pulmonary embolism after bariatric operations for morbid obesity: A 24-year retrospective analysis. Obesity Surgery Journal. 2003; 13: 819-825.

11. Escalante-Tattersfield $\mathrm{T}$, Tucker $\mathrm{O}$, Fajnwaks $P$, et al: Incidence of deep venous thrombosis in morbidly obese patients undergoing 
laparoscopic Roux-en- $\mathrm{Y}$ gastric bypass. Surg Obes Relat Dis. 2008; 4: 50-54.

12. Belle $\mathrm{SH}$, et al: Relationship of body mass index with demographic and clinical characteristics in longitudinal assessment of bariatric surgery (LABS). Surg Obes Relat Dis. 2008; 4(4): 47480. 